\title{
Analytical Solution for Different Profiles of Fin with Temperature-Dependent Thermal Conductivity
}

\author{
A. Moradi ${ }^{1}$ and H. Ahmadikia ${ }^{2}$ \\ ${ }^{1}$ Young Researchers Club, Islamic Azad University, Arak Branch, P.O. Box 38149-54688, Arak, Iran \\ ${ }^{2}$ Department of Mechanical Engineering, University of Isfahan, Isfahan 81746-73441, Iran
}

Correspondence should be addressed to A. Moradi, amirmoradi_hs@yahoo.com

Received 25 October 2010; Accepted 30 December 2010

Academic Editor: J. Jiang

Copyright (C) 2010 A. Moradi and H. Ahmadikia. This is an open access article distributed under the Creative Commons Attribution License, which permits unrestricted use, distribution, and reproduction in any medium, provided the original work is properly cited.

\begin{abstract}
Three different profiles of the straight fin that has a temperature-dependent thermal conductivity are investigated by differential transformation method (DTM) and compared with numerical solution. Fin profiles are rectangular, convex, and exponential. For validation of the DTM, the heat equation is solved numerically by the fourth-order Runge-Kutta method. The temperature distribution, fin efficiency, and fin heat transfer rate are presented for three fin profiles and a range of values of heat transfer parameters. DTM results indicate that series converge rapidly with high accuracy. The efficiency and base temperature of the exponential profile are higher than the rectangular and the convex profiles. The results indicate that the numerical data and analytical method are in agreement with each other.
\end{abstract}

\section{Introduction}

Heat transfer through fin surfaces is widely used in many industrial applications. The majority of the physical phenomena in the real world are described by nonlinear differential equations, whereas large class of these equations do not have an analytical solution. The numerical methods are widely used in solving nonlinear equations. There are some analytic methods for solving differential equations, such as Adomian decomposition method (ADM), HAM (homotopy analysis method), sinh-cosh method, homotopy perturbation method (HPM), DTM, and variational iteration method (VIM).

An analytical solution for straight fin with combined heat and mass transfer is applied by Sharqawy and Zubair [1]. They used the four different profiles for the fin and compared the temperature profile and fin efficiency for them. Sharqawy and Zubair [2] applied the analytical method for the annular fin with combined heat and mass transfer as well. 
The nonlinear similarity solution in fin equation is applied by Bokharie et al. [3]. Abbasbandy and Shivanian [4] obtained the exact analytical solution of a nonlinear equation arising in heat transfer. HAM is used by Khani et al. [5] to evaluate the analytical approximate solution and the nonlinear problem efficiency with temperature-dependent thermal conductivity and variable heat transfer coefficient. Arslanturk [6] and Rajabi [7] obtained efficiency and fin temperature distribution by ADM and the HPM with temperature-dependent thermal conductivity. An analytical method for determining of the optimum thermal design of convective longitudinal fin arrays is presented by Franco [8]. Lin and Lee [9] investigated boiling on a straight fin with linearly varying thermal conductivity.

The concept of differential transformation method was first introduced by Zhou [10] in 1986, and it was used in solving both the linear and nonlinear initial value problems in electric circuit analysis. The main advantage of this method is its direct applicability to the linear and nonlinear differential equations without requiring linearization, discretization or perturbation. Rashidi and Erfani [11] used DTM to find the fin efficiency of convective straight fins with temperature-dependent thermal conductivity. They compared the DTM results with HAM. S.-H. Chang and I.-L. Chang [12, 13] used a new algorithm for computation of one- and two-dimensional differential transform of nonlinear functions. The reduced differential transformation method for solving gas dynamic problem was used by Keskin and Oturanç [14]. Chen and Ju [15] used the differential transformation to transient advective-dispersive transport equation. Linear and nonlinear initial value problems are solved by Jang [16] with the projected differential transform method. This method can be easily applied to the initial value problem by less computational work. Hassan [17] used DTM for solving eigenvalue problems such as vibration problems.

The differential transformation method is used to solve a wide range of physical problems. This method provides a direct scheme for solving linear and nonlinear deterministic and stochastic equations without linearization and yield convergent series solution rapidly.

In this paper, we extend the application of the differential transformation method, which is based on the Taylor series expansion, to construct analytical approximate solutions of the governing equations of the straight fins with three different profiles and temperaturedependent thermal conductivity. In the previous researches, the conduction heat transfer in the rectangular fin has been studied, while the exponential and convex profiles have not been studied so far. In this paper the conduction heat transfer in these two profiles is studied and their results are compared with rectangular profile results. The temperature profile and the fin efficiency are obtained for different parameters that appear in the governing equations. Some numerical examples are presented here to illustrate the efficiency and reliability of the DTM.

\section{Fundamentals of Differential Transformation Method}

Consider the analytic function $y(t)$ in a domain $D$, where $t=t_{i}$ represent any point in it. The function $y(t)$ is represented by a power series at center $t_{i}$. The Taylor series expansion function of $y(t)$ is in the following form [18]:

$$
y(t)=\sum_{j=0}^{\infty} \frac{\left(t-t_{i}\right)^{j}}{j !}\left[\frac{d^{j} y(t)}{d t^{j}}\right]_{t=t_{i}}, \quad \forall t \in D
$$


Table 1: The fundamental operations of differential transform method.

\begin{tabular}{lc}
\hline Original function & Transformed function \\
\hline$f(x)=\alpha g(x) \pm \beta h(x)$ & $F(k)=\alpha G(k) \pm \beta H(k)$ \\
$f(x)=g(x) h(x)$ & $F(k)=\sum_{i=0}^{k} G(i) H(k-i)$ \\
$f(x)=g(x)^{(n)}$ & $F(k)=(k+1)(k+2) \cdots(k+n) G(k+n)$ \\
$f(x)=x^{n}$ & $F(k)=\delta(k-n)=\left\{\begin{array}{cc}1 & k=n \\
0 & k \neq n\end{array}\right\}$ \\
$f(x)=\exp (\alpha x)$ & $F(k)=\alpha^{k} / k !$ \\
$f(x)=(1+x)^{n}$ & $F(k)=k(k-1) \cdots(k-m-1) / k !$ \\
\hline
\end{tabular}

The particular case of (2.1) is when $t_{i}=0$ and is referred to as the Maclaurin series of $y(t)$ expressed as

$$
y(t)=\sum_{j=0}^{\infty} \frac{t^{j}}{j !}\left[\frac{d^{j} y(t)}{d t^{j}}\right]_{t=0}, \quad \forall t \in D
$$

As explained by Franco [8], differential transformation of the function $y(t)$ is defined as

$$
Y(j)=\sum_{j=0}^{\infty} \frac{H^{j}}{j !}\left[\frac{d^{j} y(t)}{d t^{j}}\right]_{t=0},
$$

where $y(t)$ is the original function and $Y(j)$ is the transformed function. The differential spectrum of $Y(j)$ is confined within the interval $t \in[0, H]$, where $H$ is a constant. The differential inverse transform of $Y(j)$ is defined as

$$
y(t)=\sum_{j=0}^{\infty}\left(\frac{t}{H}\right)^{j} Y(j)
$$

Some of the original functions and transformed functions are shown in Table 1. It is clear that the concept of differential transformation is the Taylor series expansion. For the solution with higher accuracy, more terms in the series in (2.4) should be retained.

\section{Description of the Problem}

Consider a straight fin of the length $L$, with a cross-section area $A(x)$. Fin surface is exposed to a convective environment at temperature $T_{\infty}$. The local heat transfer coefficient $h$ along the fin surface is constant, and the thermal conductivity varies with the temperature linearly. The one-dimensional energy equation can be expressed as:

$$
\frac{d}{d x}\left[k(T) A(x) \frac{d T}{d x}\right]-p h\left(T-T_{\infty}\right)=0,
$$


where $p$ is the periphery of the fin, $T_{\infty}$ is the ambient temperature and $k(T)$ is defined as

$$
k(T)=k_{b}\left[1+\lambda\left(T-T_{\infty}\right)\right]
$$

where $k_{b}$ is the fin thermal conductivity at ambient temperature and $\lambda$ is a constant.

Straight fin can be classified according to its profile as shown in Figure 1. The fin profile is defined according to variation of the fin thickness along its extended length. For example, the cross-section area of the fin may vary as

$$
A(x)=b t(x)
$$

where $b$ is the width of the fin, $t(x)$ is the fin thickness along the length. The $t(x)$ for different profiles can be defined as follows

(a) for rectangular profile

$$
t(x)=t_{b}
$$

(b) for exponential profile

$$
t(x)=t_{b} e^{a(x / L)},
$$

(c) for convex profile

$$
t(x)=t_{b}\left(\frac{x}{L}\right)^{1 / 2}
$$

by employing the following dimensionless parameters:

$$
\theta=\frac{T-T_{\infty}}{T-T_{b}}, \quad X=\frac{x}{L}, \quad N=\left(\frac{h p L^{2}}{K_{b} A_{b}}\right)^{1 / 2},
$$

where, $A_{b}$ is the base area. Thus, the energy equation for three profiles are reduced to

$$
\begin{gathered}
(1+\beta \theta) \frac{d^{2} \theta}{d X^{2}}+\beta\left(\frac{d \theta}{d X}\right)^{2}-N^{2} \theta=0, \quad \text { rectangular profile, } \\
(1+\beta \theta) e^{a X} \frac{d^{2} \theta}{d X^{2}}+a(1+\beta \theta) e^{a X} \frac{d \theta}{d X}+\beta e^{a X}\left(\frac{d \theta}{d X}\right)^{2}-N^{2} \theta=0, \quad \text { exponential profile, } \\
(1+\beta \theta) X^{1 / 2} \frac{d^{2} \theta}{d X^{2}}+\frac{(1+\beta \theta)}{2} X^{-1 / 2} \frac{d \theta}{d X}+\beta X^{1 / 2}\left(\frac{d \theta}{d X}\right)^{2}-N^{2} \theta=0, \quad \text { convex profile, }
\end{gathered}
$$




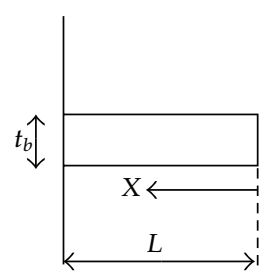

(a) Rectangular profile

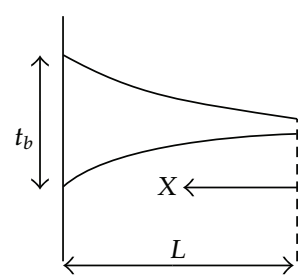

(b-1) Exponential profile $(a<0)$

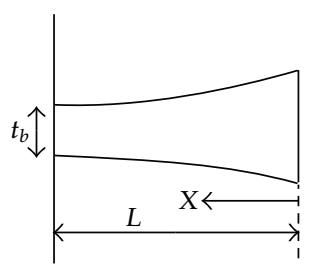

(b-2) Exponential profile $(a>0)$

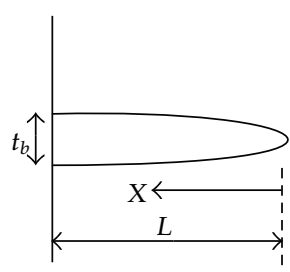

(c) Convex profile

Figure 1: Schematic of different straight fin profiles.

where, $\beta=\lambda\left(T_{b}-T_{\infty}\right)$ in which $T_{b}$ is the base temperature and fin tip is insulated. Therefore, boundary conditions for this problem are defined as follows:

$$
\begin{gathered}
X=0, \quad \frac{d \theta}{d X}=0, \\
X=1, \quad \theta=1 .
\end{gathered}
$$

\section{Solution with Differential Transformation Method}

By one-dimensional transform of (3.8)-(3.9) considered by using the related definition in Table 1, we have the following:

(a) rectangular profile

$$
\begin{aligned}
(j+1) & (j+2) \Theta(j+2)+\beta \sum_{i=0}^{j} \Theta(i)(j-i+1)(j-i+2) \Theta(j-i+2) \\
& +\beta \sum_{i=0}^{j}(i+1) \Theta(i+1)(j-i+1) \Theta(j-i+1)-N^{2} \Theta(j) \\
= & 0
\end{aligned}
$$

(b) exponential profile

$$
\begin{aligned}
& \sum_{i=0}^{j} \frac{a^{i}}{i !}(j-i+1)(j-i+2) \Theta(j-i+2) \\
& \quad+\beta \sum_{s=0}^{j} \sum_{i=0}^{j-s} \frac{a^{s}}{s !}(j-i-s+1)(j-i-s+2) \Theta(i) \Theta(j-i-s+2) \\
& \quad+a \sum_{i=0}^{j} \frac{a^{i}}{i !}(j-i+1) \Theta(j-i+1)+a \beta \sum_{s=0}^{j} \sum_{i=0}^{j-s} \frac{a^{s}}{s !}(j-i-s+1) \Theta(i) \Theta(j-i-s+1) \\
& \quad+\beta \sum_{s=0}^{j} \sum_{i=0}^{j-s} \frac{a^{s}}{s !}(i+1)(j-i-s+1) \Theta(i+1) \Theta(j-i-s+1)-N^{2} \Theta(j) \\
& =0,
\end{aligned}
$$


For convex profile, with definition $y=X^{1 / 2}$ and a substitution in (3.10), we obtain:

$$
(1+\beta \theta) \frac{d^{2} \theta}{d y^{2}}+\beta\left(\frac{d \theta}{d y}\right)^{2}-4 N^{2} y \theta=0
$$

Taking the one dimensional transform of (4.3), gives

(c) convex profile

$$
\begin{aligned}
& (j+1)(j+2) \Theta(j+2)+\beta \sum_{i=0}^{j} \Theta(i)(j-i+1)(j-i+2) \Theta(j-i+2) \\
& \quad+\beta \sum_{i=0}^{j}(i+1) \Theta(i+1)(j-i+1) \Theta(j-i+1)-4 N^{2} \sum_{i=0}^{j} \delta(i-1) \Theta(j-i) \\
& =0 .
\end{aligned}
$$

In the above equations $\Theta(j)$ is transformed function of $\Theta(X)$. The transformed boundary condition takes the form:

$$
\begin{gathered}
\Theta(1)=0, \\
\sum_{i=0}^{\infty} \Theta(i)=1 .
\end{gathered}
$$

Supposing that $\Theta(0)=\alpha$ and using (4.5) and (4.6), another value of $\Theta(i)$ for three profiles can be calculated. The value of $\alpha$ can be calculated using (4.6). Thus, we end up having the following:

(a) rectangular profile

$$
\begin{aligned}
& \Theta(2)=\frac{N^{2} \alpha}{2(1+\alpha \beta)}, \\
& \Theta(3)=0 \\
& \Theta(4)=\frac{N^{4} \alpha(1-2 \alpha \beta)}{24(1+\alpha \beta)^{3}}, \\
& \Theta(5)=0 \\
& \Theta(6)=\frac{N^{6} \alpha\left(1-16 \alpha \beta+28 \alpha^{2} \beta^{2}\right)}{720(1+\alpha \beta)^{5}}, \\
& \Theta(7)=0,
\end{aligned}
$$


(b) exponential profile

$$
\begin{aligned}
& \Theta(2)=\frac{N^{2} \alpha}{2(1+\alpha \beta)}, \\
& \Theta(3)=\frac{-a N^{2} \alpha}{3(1+\alpha \beta)}, \\
& \Theta(4)=\frac{\left(\left(N^{4} \alpha(2-4 \alpha \beta) /(2+2 \alpha \beta)^{2}\right)+\left(a^{2} N^{2} \alpha\left(18+36 \alpha \beta+18 \alpha^{2} \beta^{2}\right) /(2+2 \alpha \beta)(6+6 \alpha \beta)\right)\right)}{12(1+\alpha \beta)}
\end{aligned}
$$

(c) convex profile

$$
\begin{aligned}
& \Theta(2)=0, \\
& \Theta(3)=\frac{2 N^{2} \alpha}{3(1+\alpha \beta)}, \\
& \Theta(4)=0, \\
& \Theta(5)=0, \\
& \Theta(6)=\frac{N^{4} \alpha(0.8-1.2 \alpha \beta)}{9(1+\alpha \beta)^{3}},
\end{aligned}
$$

From the above continuing process, substituting (4.7) in (2.4) for $H=1$, we can obtain the closed form of the solution:

(a) Rectangular profile

$$
\theta(X)=\alpha+\frac{N^{2} \alpha}{2(1+\alpha \beta)} X^{2}+\frac{N^{4} \alpha(1-2 \alpha \beta)}{24(1+\alpha \beta)^{3}} X^{4}+\frac{N^{6} \alpha\left(1-16 \alpha \beta+28 \alpha^{2} \beta^{2}\right)}{720(1+\alpha \beta)^{5}} X^{6}+\cdots
$$

In order to obtain the value $\alpha$, we used (4.6). Then, we will have

$$
\theta(1)=\alpha+\frac{N^{2} \alpha}{2(1+\alpha \beta)}+\frac{N^{4} \alpha(1-2 \alpha \beta)}{24(1+\alpha \beta)^{3}}+\frac{N^{6} \alpha\left(1-16 \alpha \beta+28 \alpha^{2} \beta^{2}\right)}{720(1+\alpha \beta)^{5}}+\cdots=1 .
$$

Solving (4.11) by mathematica software gives the value of $\alpha$. For the other two profiles the same process is used to obtain the value of $\alpha$ and temperature distribution. 


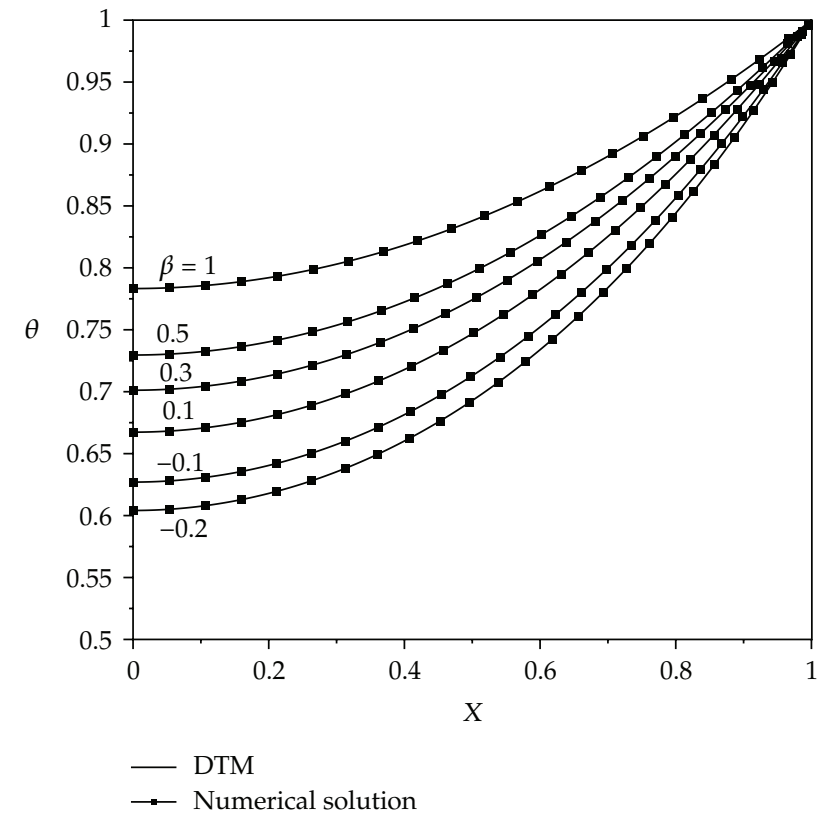

Figure 2: Temperature distribution of rectangular profile at different values of $\beta$ at $N=1$.

\section{Results and Discussion}

For assigned DTM results, we used 40 terms of the final power series. Temperature distribution for different values of $\beta$ for $N=1$ is presented for rectangular, exponential and convex profiles in Figures 2, 3, and 4, respectively. Here, the DTM results are compared to numerical data while showing a good agreement between two methods. Fourth-order RungeKutta method is applied to obtain the numerical solution. These results show that the fin tip temperature for exponential profile is greater than that of the other profiles. The exact analytical solution for fin with constant thermal conductivity $(\beta=0)$ for three profiles is calculated as follows:

(a) rectangular profile

$$
\theta(X)=\frac{e^{N X}+e^{-N X}}{e^{N}+e^{-N}}
$$

(b) exponential profile

$$
\theta(X)=\frac{\sqrt{e^{-a X}}\left(I_{1}\left[2 \sqrt{e^{-a X}} N / a\right] K_{0}[2 N / a]+I_{0}[2 N / a] K_{1}\left[2 \sqrt{e^{-a X}} N / a\right]\right)}{\sqrt{e^{-a}}\left(I_{1}\left[2 \sqrt{e^{-a}} N / a\right] K_{0}[2 N / a]+I_{0}[2 N / a] K_{1}\left[2 \sqrt{e^{-a}} N / a\right]\right)}
$$




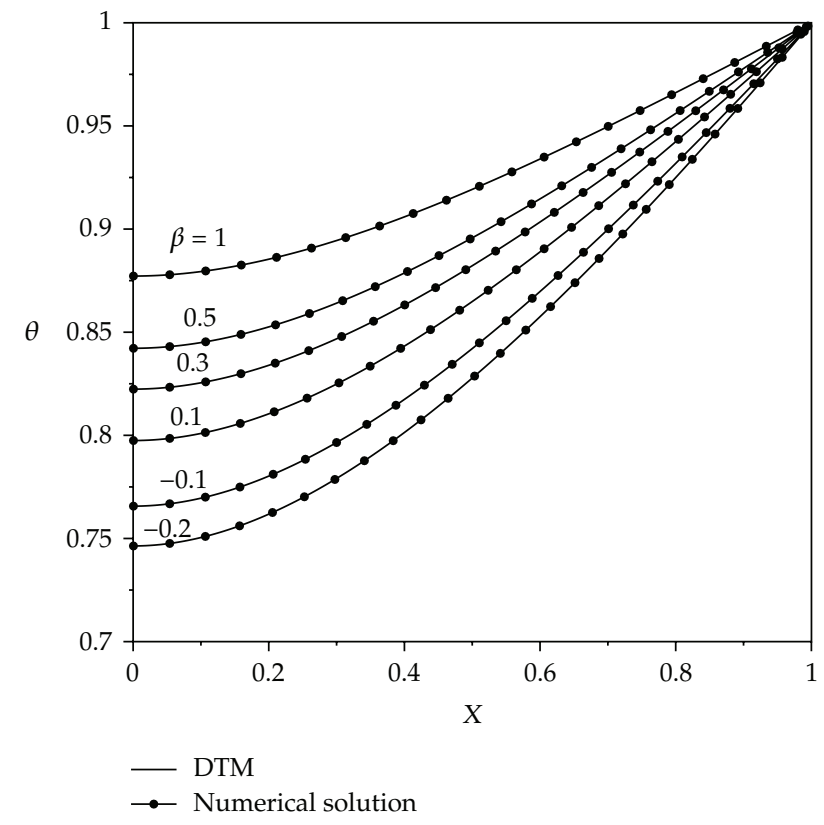

Figure 3: Temperature distribution of exponential profile $(a=1)$ for different values of $\beta$ at $N=1$.

(c) convex profile

$$
\theta[X]=\frac{((2 / 3) N)^{1 / 3} X^{1 / 4} I_{-1 / 3}\left[(4 / 3) N X^{3 / 4}\right]}{{ }_{0} \widetilde{F}_{1}\left[2 / 3,4 N^{2} / 9\right]}
$$

where $I_{n}(x)$ is the modified Bessel function of the first kind that is closely related to the Bessel function of the first kind $J_{n}(x)$ and $K_{n}(x)$ is the modified Bessel function of the second kind that is closely related to the modified Bessel function of the first kind $I_{n}(x)$ and Hankel function $H_{n}(x) .{ }_{0} \widetilde{F}_{1}$ is the regularized confluent hypergeometric function. $I_{n}(x), K_{n}(x)$ and ${ }_{0} \widetilde{F}_{1}$ are defined as

$$
\begin{gathered}
I_{n}(x)=i^{-n} J_{n}(i x)=e^{-n \pi i / 2} J_{n}\left(x e^{i \pi / 2}\right), \\
K_{n}(x)=\frac{1}{2} \pi i^{n+1} H_{n}(i x)=\frac{\pi}{2} \frac{I_{-n}(x)-I_{n}(x)}{\sin (n \pi)}, \\
{ }_{0} \tilde{F}_{1}(b ; x)=\sum_{k=0}^{\infty} \frac{x^{k}}{\Gamma(b+k) k !}
\end{gathered}
$$

where $\Gamma$ is the Gamma function.

The comparison between the exact and DTM results for three profiles at $\beta=0$ and $N=1$ is shown in Table 2 . This comparison shows that DTM results are very close to the exact analytical solution, so that we can conclude that DTM is a proper method for solving linear 


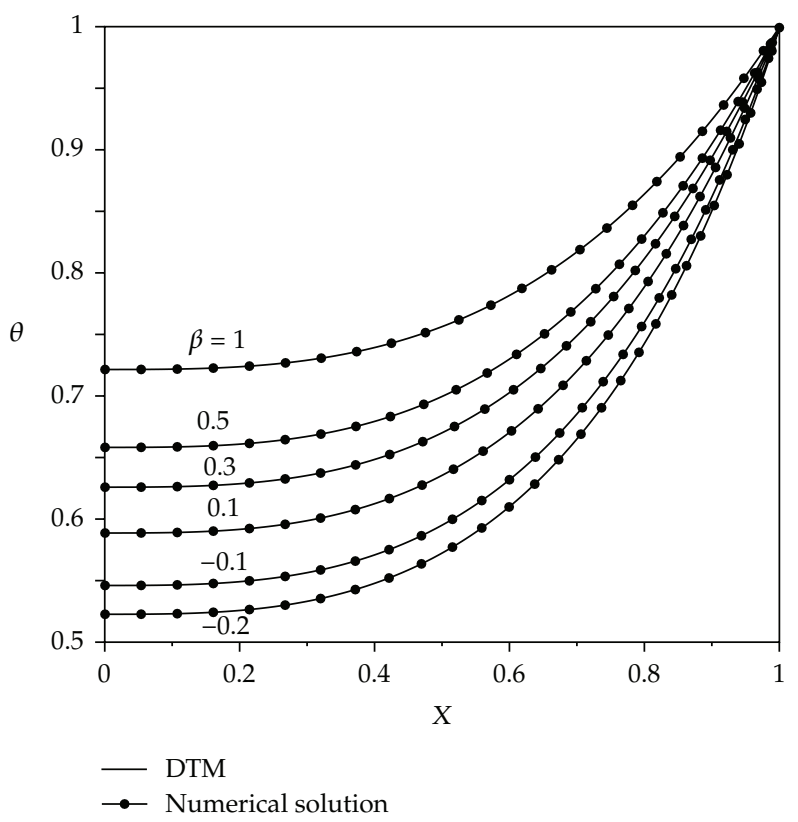

Figure 4: Temperature distribution of convex profile for different values of $\beta$ at $N=1$.

and nonlinear equations. The comparison between present results and other reported results for rectangular profile for $\beta=0$ and $N=1$ is shown in Table 3. There exists an indirect relation between the tip temperature $\Theta(0)=\alpha$ rise and the values of $N$, because when $N$ increases, the convective heat transfer rate increases, so that fin tip temperature decreases. For investigating the effect of the different profiles on the straight fin performance, the temperature profile is presented for different values of $N$ at $\beta=1$ for three profiles, see Figure 5 . From the results it can be concluded that, with decreasing $\beta$, the fin base temperature decreases for any profile in the straight fin.

The most important characteristics of the fins that are studied in the engineering heat transfer problems are the fin efficiency and fin effectiveness. If we define the fin efficiency $\eta$ in a usual way as the ratio of the actual heat transfer rate through the base of a fin to the ideal heat flow rate if the whole fin was the same temperature as the base of the fins, therefore, the fin efficiency can be expressed as

$$
\eta=\frac{\int_{0}^{L} \operatorname{Ph}\left(T-T_{\infty}\right) d x}{\operatorname{PLh}\left(T_{b}-T_{\infty}\right)}=\int_{0}^{1} \theta d X .
$$

Fin efficiency for several assigned values of $\beta$ is shown in Figure 6. The results show that, for positive values of $\beta$, the efficiency is greater with respect to negative values of $\beta$. Likewise the efficiency for exponential profile is greater than for other profiles, because at the exponential profile, the fin base temperature is greater than that of the other profiles. Of course it should be mentioned that the exponential profile with positive power $(a>0)$ has a higher efficiency. The temperature distribution for the different values of $a$ (exponential parameter) is shown at $N=1$ and compared with other results in Figure 7 . 
Table 2: Comparison between exact and DTM results for three profiles at $\beta=0$ and $N=1$.

\begin{tabular}{lcccccc}
\hline \multirow{2}{*}{$X$} & \multicolumn{2}{c}{ Convex profile } & \multicolumn{2}{c}{ Exponential } & profile $(a=1)$ & \multicolumn{2}{c}{ Rectangular profile } \\
& Exact & DTM & Exact & DTM & Exact & DTM \\
\hline 0 & 0.56797321 & 0.56797323 & 0.78267175 & 0.78267175 & 0.648054274 & 0.648054274 \\
0.1 & 0.5799982 & 0.579997732 & 0.78633664 & 0.786336641 & 0.651297246 & 0.651297246 \\
0.2 & 0.60224731 & 0.602246515 & 0.796427754 & 0.796427754 & 0.66105862 & 0.66105862 \\
0.3 & 0.63156711 & 0.631567222 & 0.81176631 & 0.81176631 & 0.677436092 & 0.677436091 \\
0.4 & 0.66704223 & 0.667041545 & 0.831369483 & 0.831369483 & 0.700593571 & 0.700593571 \\
0.5 & 0.70828235 & 0.708281911 & 0.85441111 & 0.854411111 & 0.730762826 & 0.730762826 \\
0.6 & 0.75514423 & 0.755143954 & 0.880191752 & 0.880191753 & 0.768245801 & 0.768245801 \\
0.7 & 0.80762463 & 0.807623882 & 0.908115721 & 0.908115721 & 0.813417638 & 0.813417638 \\
0.8 & 0.86581241 & 0.865811512 & 0.937673304 & 0.937673304 & 0.866730433 & 0.866730433 \\
0.9 & 0.92986609 & 0.929865636 & 0.968426886 & 0.968426886 & 0.928717757 & 0.928717757 \\
1 & 1 & 1 & 1 & 1 & 1 & 1 \\
\hline
\end{tabular}

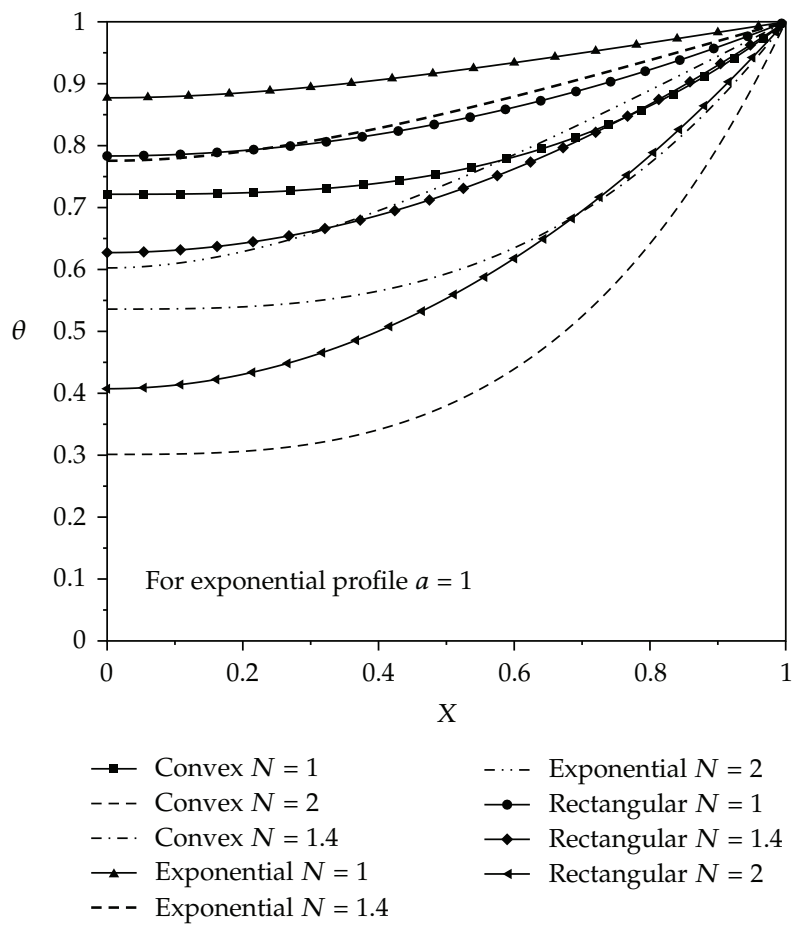

Figure 5: Temperature profile for different values of $N$ at $\beta=1$ and three profiles of the straight fin.

One of the other characteristics of the fins studied in engineering problems is the fin base heat transfer rate which can be expressed as $Q_{b}=d \theta(1) / d X$. The variation of the $Q_{b}$ with $N$ for the two assigned values of $\beta$ is shown in Figure 8 . The results show that for, smaller values of $\beta$, the value of $Q_{b}$ is greater than for larger values of $\beta$.

The results show that the straight fins with exponential profile have higher performance and efficiency in comparison with other profiles. However, in most industrial applications the rectangular profile is used due to its easy construction of rectangular fins. The fin with convex profile has a minimum efficiency and performance for industrial 
Table 3: Comparison between present and other reported results for rectangular fin for $\beta=0$ and $N=0.5$.

\begin{tabular}{lccc}
\hline$X$ & DTM & ADM [6] & HPM [7] \\
\hline 0 & 0.886818884 & 0.886819 & 0.886819 \\
0.1 & 0.887927639 & 0.887928 & 0.887928 \\
0.2 & 0.891256675 & 0.891257 & 0.891257 \\
0.3 & 0.896814317 & 0.896815 & 0.896814 \\
0.4 & 0.904614462 & 0.904615 & 0.904614 \\
0.5 & 0.914676614 & 0.914677 & 0.914677 \\
0.6 & 0.927025935 & 0.927026 & 0.927026 \\
0.7 & 0.941693303 & 0.941694 & 0.941693 \\
0.8 & 0.958715394 & 0.958716 & 0.958715 \\
0.9 & 0.978134774 & 0.978135 & 0.978135 \\
1 & 1 & 1 & 1 \\
\hline
\end{tabular}

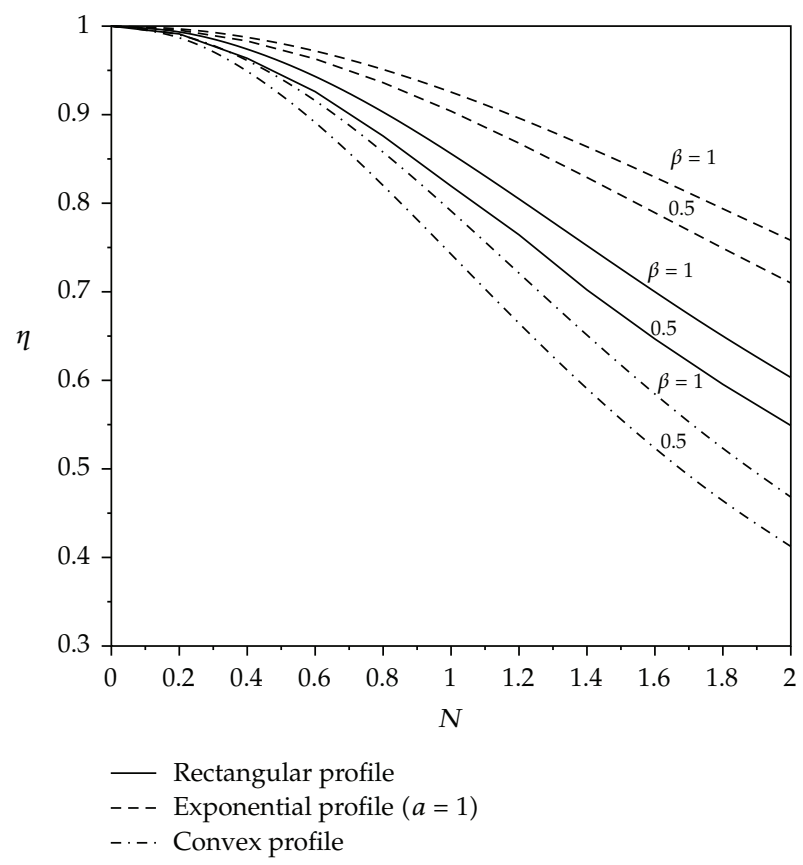

Figure 6: The variation of $\eta$ versus $N$ for two assigned values of $\beta$.

and engineering applications. In the previous researches, the exponential profile is not investigated since Sharqawy and Zubair [1] presented an analytical solution for the rectangular, triangular, concave and convex profile for the straight semiwet fin with constant thermal conductivity. They showed that the rectangular profile has a higher performance than other profiles. But, in the present research it is shown that the efficiency for exponential profile (with positive power) is even greater than that for rectangular profile.

Results show that the variable thickness at the straight fin is very important characteristic in heat conduction problems. Also, the thermal conductivity variation has direct effect on the temperature distribution and characteristics of the fin such as fin efficiency, fin base heat transfer rate, and fin effectiveness. 


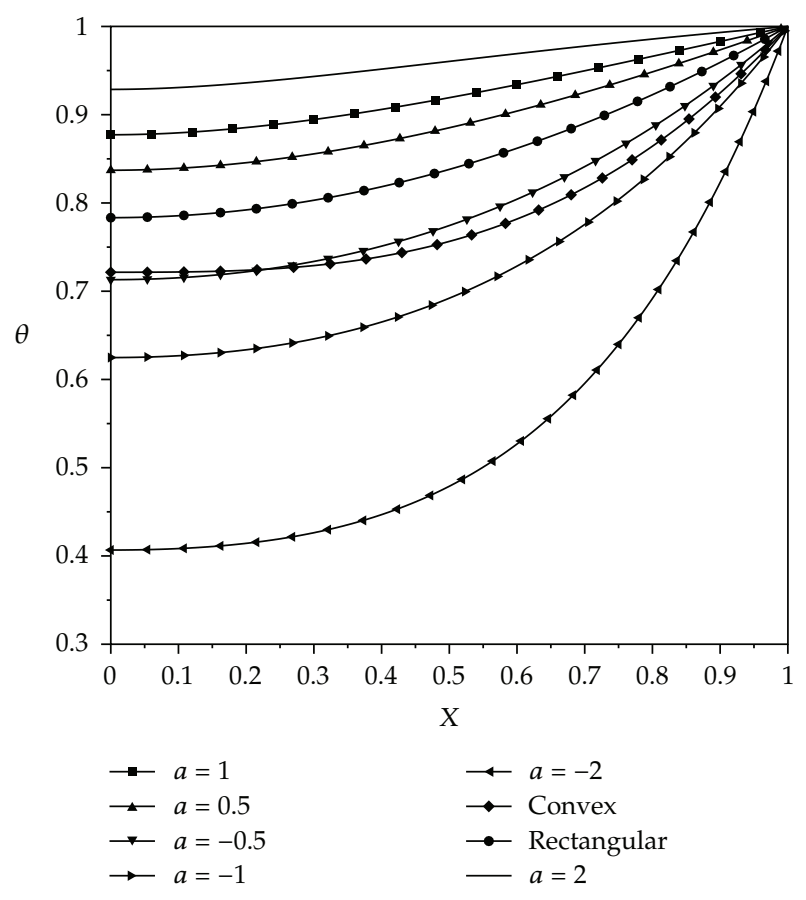

Figure 7: Temperature distribution of exponential profile with different parameter $a$ at $N=1$.

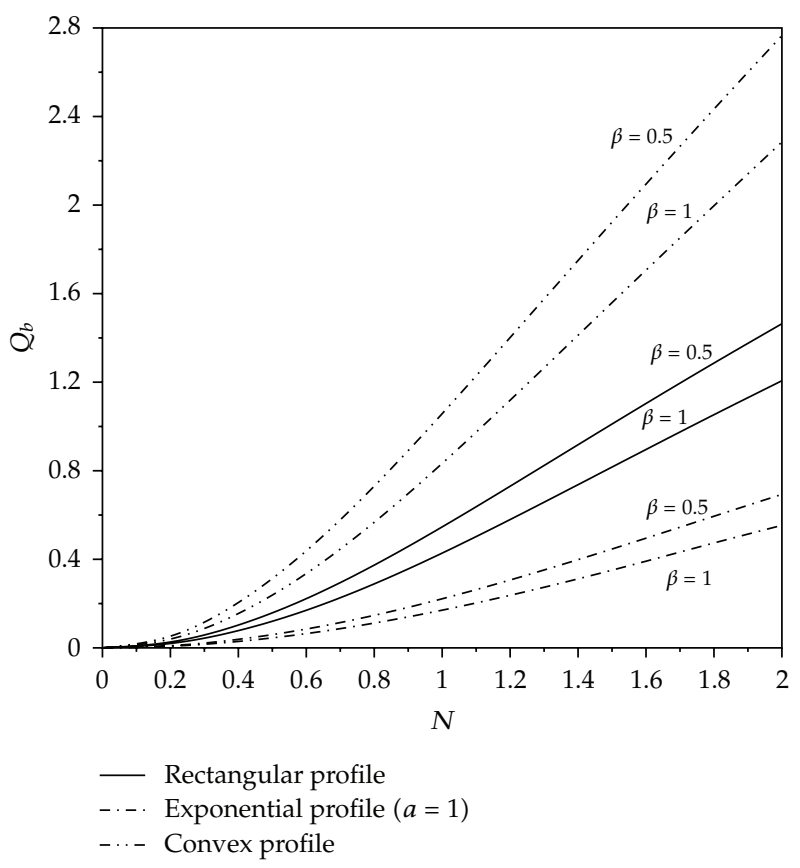

Figure 8: The variations of $Q_{b}$ with $N$ for several assigned values of $\beta$. 


\section{Conclusion}

The differential transformation method (DTM) was applied for solving heat conduction problem in the fin with different profiles and temperature-dependent thermal conductivity. This method has been applied for the linear and nonlinear differential equations. This method is an infinite power series form and has high accuracy and fast convergence. To validate the analytical results, DTM results are compared with numerical data obtained using the fourthorder Runge-Kutta method. The fin efficiency and heat transfer rate can be easily obtained from the explicit form of the temperature profile. It is shown that differential transformation method has a very fast convergency, as well as being a precise and cost-efficient tool for solving the efficiency of the fin with variable thermal conductivity. Results show that exponential profile (with positive power) has a higher performance than other profiles in any thermal conductivity condition. In general, DTM has a good approximate analytical solution for the linear and nonlinear engineering problems without any assumption and linearization.

\section{Nomenclature}

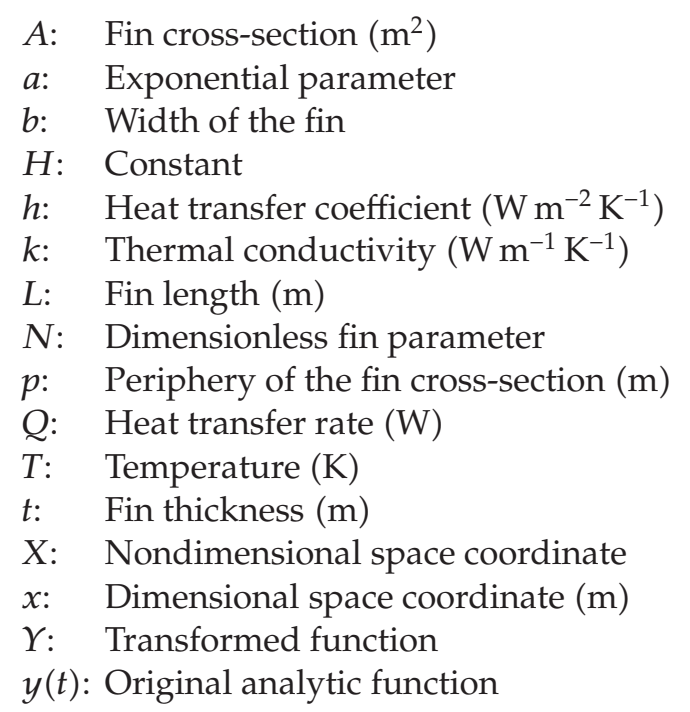

\section{Greek Symbols}

$\alpha$ : Fin base temperature

$\beta$ : Thermal expansion coefficient $\left(\mathrm{K}^{-1}\right)$

$\eta$ : Fin efficiency

$\lambda$ : Dimensional constant $\left(\mathrm{K}^{-1}\right)$

$\Theta$ : Transformed temperature

$\theta$ : Dimensionless temperature

\section{Subscripts}

$b$ : Fin base

$\infty$ : Ambient property. 


\section{Acknowledgment}

The authors thank anonymous reviewers for their valuable comments.

\section{References}

[1] M. H. Sharqawy and S. M. Zubair, "Efficiency and optimization of straight fins with combined heat and mass transfer-an analytical solution," Applied Thermal Engineering, vol. 28, no. 17-18, pp. 2279 2288, 2008.

[2] M. H. Sharqawy and S. M. Zubair, "Efficiency and optimization of an annular fin with combined heat and mass transfer-an analytical solution," International Journal of Refrigeration, vol. 30, no. 5, pp. 751-757, 2007.

[3] A. H. Bokhari, A. H. Kara, and F. D. Zaman, "A note on a symmetry analysis and exact solutions of a nonlinear fin equation," Applied Mathematics Letters, vol. 19, no. 12, pp. 1356-1360, 2006.

[4] S. Abbasbandy and E. Shivanian, "Exact analytical solution of a nonlinear equation arising in heat transfer," Physics Letters. A, vol. 374, no. 4, pp. 567-574, 2010.

[5] F. Khani, M. A. Raji, and H. H. Nejad, "Analytical solutions and efficiency of the nonlinear fin problem with temperature-dependent thermal conductivity and heat transfer coefficient," Communications in Nonlinear Science and Numerical Simulation, vol. 14, no. 8, pp. 3327-3338, 2009.

[6] C. Arslanturk, "A decomposition method for fin efficiency of convective straight fins with temperature-dependent thermal conductivity," International Communications in Heat and Mass Transfer, vol. 32, no. 6, pp. 831-841, 2005.

[7] A. Rajabi, "Homotopy perturbation method for fin efficiency of convective straight fins with temperature-dependent thermal conductivity," Physics Letters. Section A, vol. 364, no. 1, pp. 33-37, 2007.

[8] A. Franco, "An analytical method for the optimum thermal design of convective longitudinal fin arrays," Heat and Mass Transfer, vol. 45, no. 12, pp. 1503-1517, 2009.

[9] W. W. Lin and D. J. Lee, "Boiling on a straight pin fin with variable thermal conductivity," Heat and Mass Transfer, vol. 34, no. 5, pp. 381-386, 1999.

[10] J. K. Zhou, Differential Transformation Method and Its Application for Electrical Circuits, Huazhong University Press, Wuhan, China, 1986.

[11] M. M. Rashidi and E. Erfani, "New analytical method for solving Burgers' and nonlinear heat transfer equations and comparison with HAM," Computer Physics Communications, vol. 180, no. 9, pp. 1539 1544, 2009.

[12] S.-H. Chang and I.-L. Chang, "A new algorithm for calculating one-dimensional differential transform of nonlinear functions," Applied Mathematics and Computation, vol. 195, no. 2, pp. 799-805, 2008.

[13] S.-H. Chang and I.-L. Chang, "A new algorithm for calculating two-dimensional differential transform of nonlinear functions," Applied Mathematics and Computation, vol. 215, no. 7, pp. 2486-2494, 2009.

[14] Y. Keskin and G. Oturanç, "Application of reduced differential transformation method for solving gas dynamic equation," International Journal of Contemporary Mathematical Sciences, vol. 22, no. 22, pp. 1091-1096, 2010.

[15] C.-K. Chen and S.-P. Ju, "Application of differential transformation to transient advective-dispersive transport equation," Applied Mathematics and Computation, vol. 155, no. 1, pp. 25-38, 2004.

[16] B. Jang, "Solving linear and nonlinear initial value problems by the projected differential transform method," Computer Physics Communications, vol. 181, no. 5, pp. 848-854, 2010.

[17] I. H. Hassan, "On solving some eigenvalue problems by using a differential transformation," Applied Mathematics and Computation, vol. 127, no. 1, pp. 1-22, 2002.

[18] A. A. Joneidi, D. D. Ganji, and M. Babaelahi, "Differential Transformation Method to determine fin efficiency of convective straight fins with temperature dependent thermal conductivity," International Communications in Heat and Mass Transfer, vol. 36, no. 7, pp. 757-762, 2009. 


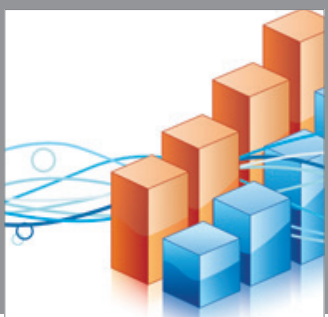

Advances in

Operations Research

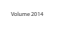

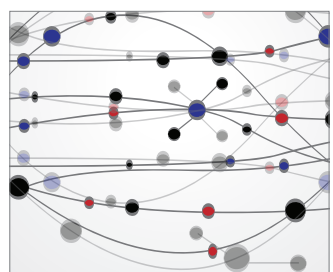

\section{The Scientific} World Journal
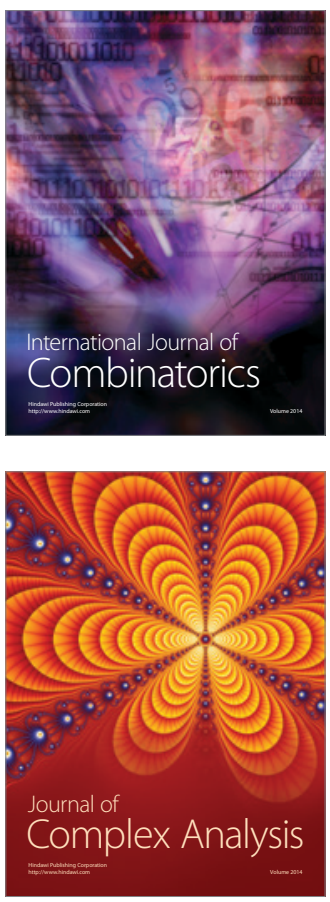

International Journal of

Mathematics and

Mathematical

Sciences
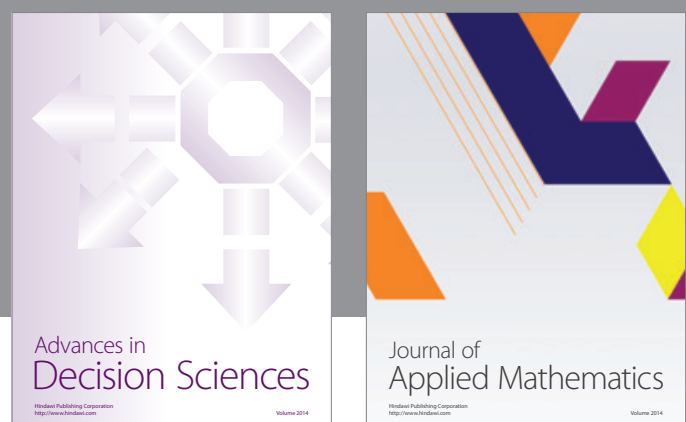

Journal of

Applied Mathematics
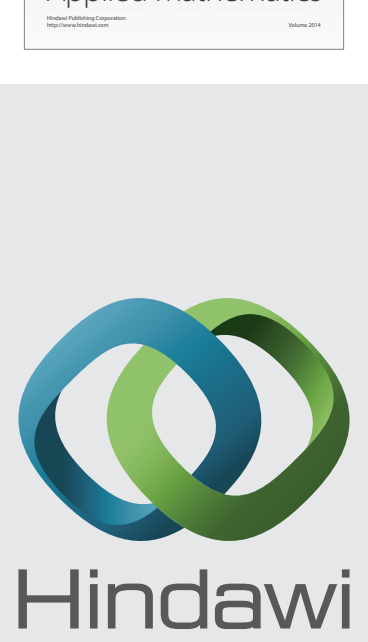

Submit your manuscripts at http://www.hindawi.com
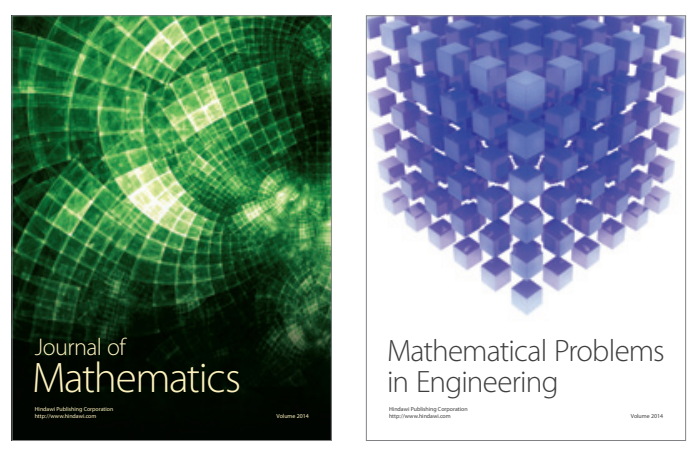

Mathematical Problems in Engineering
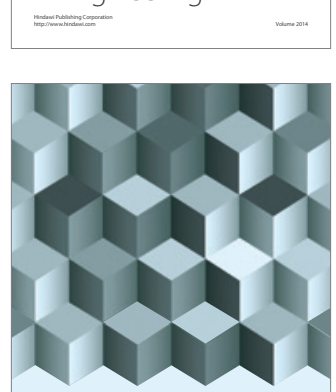

Journal of

Function Spaces
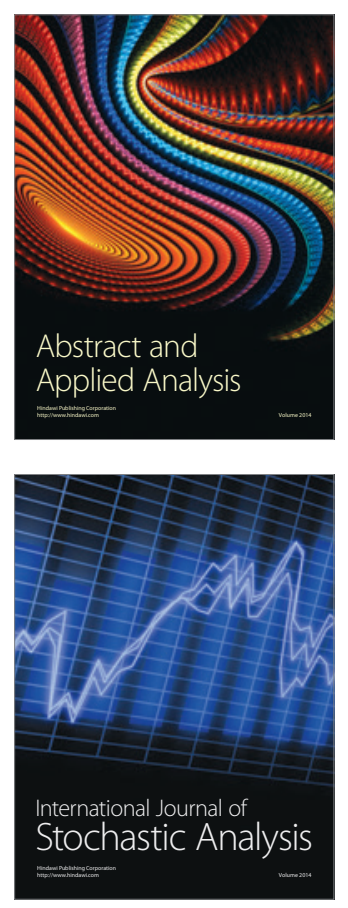

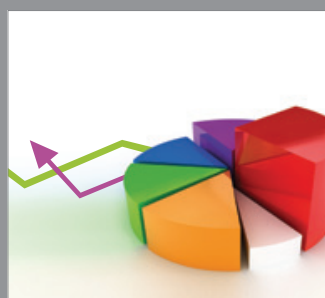

ournal of

Probability and Statistics

Promensencen
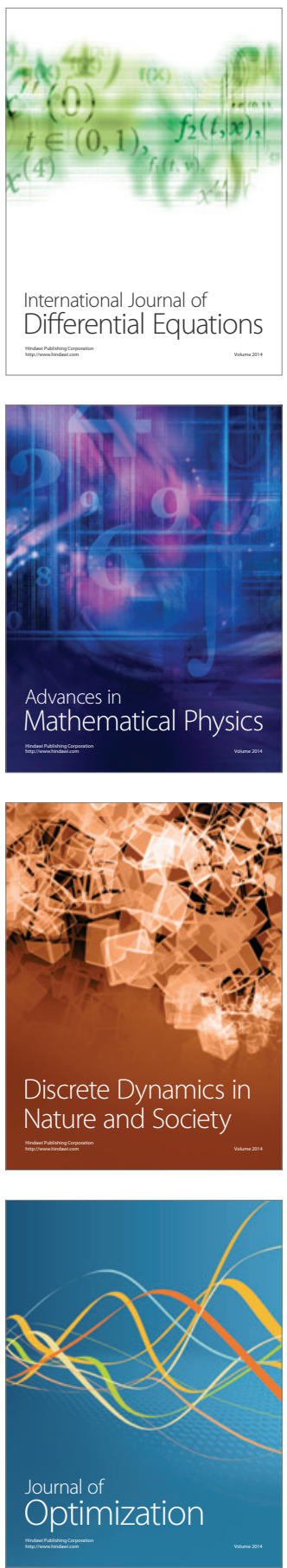